\title{
PUNCH-CARD MACHINE METHODS APPLIED TO THE SOLUTION OF THE TORSION PROBLEM*
}

\author{
BY \\ STEFAN BERGMAN \\ Harvard University
}

Introduction. Many problems in Engineering and Physics can be reduced to boundary value problems, i.e. to problems involving the determination of a function which satisfies a given partial differential equation inside a domain and assumes perscribed values on the boundary of this domain. Despite the fact that the solutions of many such problems have been known "in principle," their actual evaluation has required such a great amount of computation that it has only been possible to carry out calculations of this kind in a few simple cases.

The creation of modern computational devices such as punch-card machines, IBM-Harvard and Bell Telephone Laboratory machines, the ENIAC computer, etc., which can carry out rather extensive computations automatically, has changed the picture completely. However, these machines exist only to implement theoretical methods, and the reduction of these methods to a form whereby the machine can "take hold" represents a problem in itself.

In previous publications the author developed certain theoretical methods (the "method of orthogonal functions" and the "method of particular solutions") for solving boundary-value problems. The present paper illustrates the application of orthogonal functions to the solution of Laplace's equation $\left(\partial^{2} \phi / \partial x^{2}\right)+\left(\partial^{2} \phi / \partial y^{2}\right)=0$ through the use of punch-card machines.

1. Formulation of a problem in elasticity. The present paper is concerned with a method of solving the torsion problem for a bar of uniform cross-section.

Let $x, y, Z$ denote rectangular coordinates, the axis of $Z$ being perpendicular to the cross-section of the beam. ${ }^{1}$ According to Saint Venant the components $u, v, w$ of the displacement vector are given by the expressions

$$
u=-\tau y Z, \quad v=\tau x Z, \quad w=\frac{1}{2} \tau G(x, y)
$$

and the components of the stresses, $X_{Z}$ and $Y_{Z}$ by

$$
X_{Z}=\mu \tau\left[\frac{1}{2}(\partial H / \partial y)-y\right], \quad Y_{Z}=\mu \tau\left[x-\frac{1}{2}(\partial H / \partial y)\right] .
$$

Here $\tau$ is the angle of twist per unit length, $\mu$ is the modulus of rigidity, $G(x, y)$ and $H(x, y)$ are conjugate harmonic functions.

On the boundary of the cross-section, the function $H(x, y)$ assumes the values $x^{2}+y^{2}$. These conditions determine $G$ and $H$ uniquely within an additive constant for $G$.

The torsion problem is thus reduced to the "first boundary value problem" of potential theory. We shall describe a method of solving it. As an illustrative example, we shall determine the function $H$ in the case of the domain indicated in Fig. 1.

* Received June 14, 1946.

1 This coordinate is denoted by $Z$ rather than $z$ in order that this last symbol can be used to designate the complex variable $z=x+i y$. 
If one of the functions, $G$ or $H$, is known, the determination of the other function is very simple. Despite the fact that for the displacement components we need $G$ in our example, we determine the function $H$, since its values are given on the boundary and the reader can directly estimate how much our approximate solution differs from the required values on the boundary.

2. The method of orthogonal polynomials. In this paper the method of orthogonal polynomials will be used for the determination of the function $H$ defined above. The mathematical ideas underlying this method have been developed by the author in [1],* pp. 57-59 and supplementary Note No. II, [2] and in other papers which are listed in these references. However, the necessary computations are very involved;

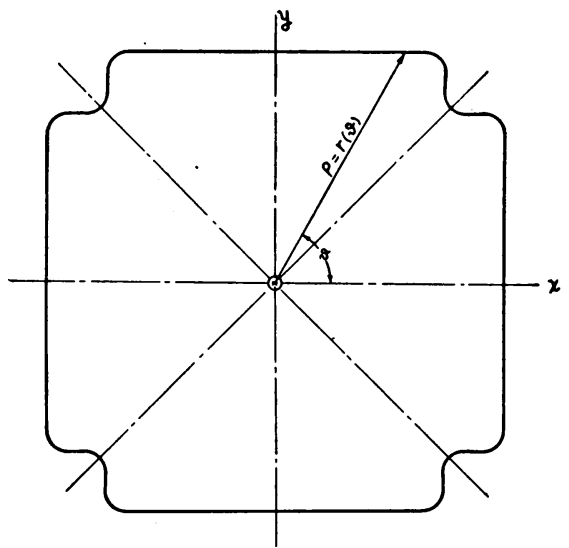

Fig. 1. The cross section $B$ of the bar. the aim of the present paper is to indicate how they can be performed with the use of punchcard machines.

There exist various methods for solving the boundary-value problem of Laplace's equation. The method of orthogonal functions has the following advantages.

1) One can obtain the solution in a form in which the dependence on various parameters is evident; in particular one can obtain a solution not only for one fixed domain but for a whole family of domains which depend upon one of several parameters. For instance, in the case of the domain indicated in Fig. 1, one can obtain formulas involving the radius of curvature at the corners and thus investigate how this radius influences the stress distribution.

2) In order to solve the boundary-value problem, the bulk of the computation, i.e. the determination of orthogonal functions, need be performed only once. As soon as a set of orthogonal functions for a domain $B$ is known the harmonic function which assumes given boundary values or given values of the normal derivative on the boundary can be easily determined.

3) The method is very general; it can be easily extended to various other problems, e.g. to problems in elasticity as well as to problems involving linear differential equations of elliptic type with non-constant coefficients (see Sec. 8).

Let $z=x+i y,(x, y$ real $)$, and let $\phi^{(n+1)}(z)$ denote the polynomial

$$
\phi^{(n+1)}(z)=D_{n+1}(z)\left[E_{n} E_{n+1}\right]^{-1 / 2}, \quad n=0,1,2, \cdots
$$

where $D_{n+1}$ and $E_{n+1}$ are the determinants

$$
D_{n+1}(z)=\left|\begin{array}{ccccc}
F_{0,0} & F_{0,1} & \cdots & F_{0, n-1} & 1 \\
F_{1,0} & F_{1,1} & \cdots & F_{1, n-1} & z \\
\cdot & \cdot & \cdots & . & . \\
. & \cdot & \cdots & . & . \\
F_{n, 0} & F_{n, 1} & \cdots & F_{n, n-1} & z^{n}
\end{array}\right|, \quad E_{n+1}=\left|\begin{array}{ccccc}
F_{0,0} & F_{0,1} & \cdots & F_{0, n-1} & F_{0, n} \\
\cdot & \cdot & \cdots & \cdot & \cdot \\
\cdot & \cdot & \cdots & . & . \\
\cdot & \cdot & \cdots & . & . \\
F_{n, 0} & F_{n, 1} & \cdots & F_{n, n-1} & F_{n, n}
\end{array}\right|
$$

respectively, and

\footnotetext{
* Numbers in brackets refer to the bibliography at the end of this paper.
} 


$$
F_{p q}=\iint_{B} z^{p} \bar{z}^{q} d x d y, \quad \bar{z}=x-i y .
$$

The set of polynomials $\left\{\phi^{(n)}(z)\right\}, n=1,2,3, \cdots$, constitutes an orthonormal set over the domain $B$, i.e.,

$$
\iint_{B} \phi^{(n)} \overline{\phi^{(m)}} d x d y=\left\{\begin{array}{lll}
1 & \text { if } & n=m \\
0 & \text { if } & n \neq m .
\end{array}\right.
$$

Now let

$$
\Phi^{(n)}(z)=\int_{0}^{z} \phi^{(n)}(z) d z
$$

and let the real and imaginary parts of $\Phi^{(n)}(z)$ be designated as $\psi_{n}$ and $\theta_{n}$, respectively, so that

$$
\Phi^{(n)}(z)=\psi_{n}(x, y)+i \theta_{n}(x, y) .
$$

Note that $\Phi^{(n)}(z)$ is a polynomial of degree $n$ in $z$, and that $\psi_{n}$ and $\theta_{n}$ are polynomials of degree $n$ in $x$ and $y$.

As is well-known, both $\psi_{n}$ and $\theta_{n}$ are harmonic functions. They possess the following property. Let $H(x, y)$ be a function which is harmonic in $B$ and continuous on the boundary $C$ of $B$. Let $s$ denote the length along the boundary $C$ measured from some fixed point, so that the boundary values for $H(x, y)$ may be given in the form $H(x, y)=f(s)$ on $C$. The function $H(x, y)$ and its conjugate $G(x, y)$ can be expressed in terms of prescribed boundary values $f(s)$ and the functions $\psi_{n}, \theta_{n}$ in the following form:

$$
\begin{aligned}
& H(x, y)=c_{1}+\sum_{\nu=1}^{\infty}\left[\psi_{\nu}(x, y) \int_{C} f(s) d \theta_{\nu}(s)-\theta_{\nu}(x, y) \int_{C} f(s) d \psi_{\nu}(s)\right], \\
& G(x, y)=c_{2}+\sum_{\nu=1}^{\infty}\left[\theta_{\nu}(x, y) \int_{C} f(s) d \theta_{\nu}(s)+\psi_{\nu}(x, y) \int_{C} f(s) d \psi_{\nu}(s)\right],
\end{aligned}
$$

where $c_{k}$ are constants. The proof of this statement may be found in [1], p. 58 .

In any practical case, the infinite summation (2.6) must be approximated by a summation over a sufficiently large number of functions, i.e., (2.6a) must be replaced $\mathrm{by}^{2}$

$$
H_{N}(x, y)=c_{N}+\sum_{\nu=1}^{N}\left[\psi_{\nu}(x, y) \int_{C} f(s) d \theta_{\nu}(s)-\theta_{\nu}(x, y) \int_{C} f(s) d \psi_{\nu}(s)\right],
$$

where $N$ is a positive integer. For increasing $N$ the accuracy of the approximation will constantly improve. In the present case, we shall take $N=8$.

It is thus seen that the whole problem of solving the boundary-value problem is reduced to the performance of the following computations.

(i) Evaluation of the integrals

2 In the following some quantities, e.g. $\theta_{\nu}$ and $\psi_{\nu}$, are considered as functions of different variables. In passing from one variable to another, new symbols should be introduced, since $\theta_{\nu}$ and $\psi_{\nu}$ are different functions of their respective arguments. For instance

$$
\theta_{\nu}^{(1)}(s) \equiv \theta_{\nu}[x(s), y(s)] .
$$

For the sake of brevity the superscript (or the introduction of new symbols) will be omitted and the functions will always be denoted by $\theta_{\nu}, \psi_{\nu}$, etc., irrespective of the arguments. 


$$
\iint_{B} z^{p} \overline{\tilde{z}}^{q} d x d y
$$

(ii) Computation of the determinants appearing in equation (2.1).

(iii) Evaluation of the coefficients

$$
\int_{C} f(s) d \theta_{\nu}(s), \quad \int_{C} f(s) d \psi_{\nu}(s) ;
$$

(iv) Determination of the sum appearing in the right hand side of (2.7), i.e. of $H_{N}$ for a sufficiently large $N$, and the determination of the first partial derivatives $\partial H_{N} / \partial x$ and $\partial H_{N} / \partial y$.

Using an illustrative example we shall describe in the following how each of these operations can be carried out on punch-card machines.

3. Evaluation of the integrals $F_{p q}$. In this paper we consider a bar with the crosssection shown in Fig. 1. This cross section possesses 8-fold symmetry.

$B$ is a star-domain; i.e., its boundary $C$ can be represented in polar coordinates $(\rho, \vartheta)$ in the form

$$
\rho=r(\vartheta) \quad(0 \leqq \vartheta \leqq 2 \pi) .
$$

The values of $r(\vartheta), \vartheta=0,1^{\circ}, 2^{\circ}, \cdots, 45^{\circ}$ are indicated in the second column of Table 1.

TABLE 1: The values of $\vartheta, r(\vartheta), r^{2}(\vartheta), H_{8}(r(\vartheta), \vartheta)-c_{8}$ and of $H_{8}(r(\vartheta), \vartheta)$ on the boundary $C$ of $B$.

\begin{tabular}{r||c|c|c|c}
\hline \hline$\vartheta$ & $r(\vartheta)$ & $r^{2}(\vartheta)$ & $H_{8}[r(\vartheta), \vartheta]-c_{8}$ & $H_{8}[r(\vartheta), \vartheta]$ \\
\hline 0 & 2.722 & 7.41 & -1.309 & 7.183 \\
2 & 2.723 & 7.420 & -1.295 & 7.197 \\
4 & 2.728 & 7.447 & -1.249 & 7.243 \\
6 & 2.737 & 7.491 & -1.174 & 7.318 \\
8 & 2.749 & 7.557 & -1.070 & 7.422 \\
10 & 2.764 & 7.640 & -.937 & 7.555 \\
12 & 2.783 & 7.745 & -.779 & 7.713 \\
14 & 2.805 & 7.874 & -.595 & 7.897 \\
16 & 2.831 & 8.020 & -.389 & 8.103 \\
18 & 2.862 & 8.191 & -.163 & 8.329 \\
20 & 2.896 & 8.393 & .078 & 8.570 \\
22 & 2.936 & 8.620 & .330 & 8.822 \\
24 & 2.979 & 8.880 & .586 & 9.078 \\
26 & 3.028 & 9.169 & .840 & 9.332 \\
28 & 3.083 & 9.504 & 1.085 & 9.577 \\
30 & 3.143 & 9.878 & 1.308 & 9.800 \\
32 & 3.210 & 10.304 & 1.498 & 9.990 \\
34 & 3.276 & 10.732 & 1.629 & 10.121 \\
35 & 3.291 & 10.837 & 1.639 & 10.131 \\
36 & 3.291 & 10.837 & 1.610 & 10.102 \\
37 & 3.273 & 10.719 & 1.553 & 10.045 \\
38 & 3.236 & 10.476 & 1.477 & 9.969 \\
39 & 3.179 & 10.106 & 1.388 & 9.880 \\
40 & 3.122 & 9.691 & 1.301 & 9.793 \\
41 & 3.049 & 9.303 & 1.228 & 9.720 \\
42 & 2.990 & 8.940 & 1.163 & 9.655 \\
43 & 2.941 & 8.655 & 1.113 & 9.605 \\
44 & 2.912 & 8.486 & 1.085 & 9.577 \\
45 & 2.903 & 8.428 & 1.075 & 9.567 \\
\hline
\end{tabular}


The double integral $F_{m n}$ can be replaced by a single integral. Indeed,

$$
\begin{aligned}
F_{m n}= & \int_{0}^{2 \pi} \int_{0}^{r(\vartheta)} \rho^{m+n+1} e^{i(n-m) \vartheta} d \rho d \vartheta \\
= & \frac{1}{m+n+2}\left\{\int_{0}^{2 \pi}[r(\vartheta)]^{m+n+2} \cos (n-m) \vartheta d \vartheta\right. \\
& \left.+i \int_{0}^{2 \pi}[r(\vartheta)]^{m+n+2} \sin (n-m) \vartheta d \vartheta\right\} .
\end{aligned}
$$

It follows from the aforementioned symmetry that many of the coefficients $F_{m n}$ vanish. Indeed, $F_{m n}=0$ unless $|m-n|=0,4,8, \cdots$. Also, even if $F_{m n}$ does not vanish, it can be shown that the imaginary part vanishes, so that

$$
F_{m n}=F_{n m}=\frac{1}{m+n+2} \int_{0}^{2 \pi}[r(\vartheta)]^{m+n+2} \cos (m-n) \vartheta d \vartheta .
$$

If we let $m+n+2=s$ and $m-n=q$, and take account of the symmetry once again, it is easily seen that

$$
F_{m n}=F_{n m}=(8 / s) \int_{0}^{\pi / 4}[r(\vartheta)]^{s} \cos q \vartheta d \vartheta .
$$

Taking account of the relationship between degrees and radians, and replacing the above integral by a summation, we get the approximation:

$$
\begin{aligned}
F_{m n}=F_{n m} \approx(8 / s) \cdot(\pi / 180)\left\{\frac{1}{2}[r(\vartheta)]^{s}+\frac{1}{2}\left[r\left(45^{\circ}\right)\right]^{s} \cos (q \pi / 4)\right. \\
\\
\left.+\sum_{\nu=1}^{44}\left[r\left(\nu^{\circ}\right)\right]^{s} \cos \left(q \pi \nu^{\circ} / 180\right)\right\} .
\end{aligned}
$$

The above sum can be conveniently evaluated by using the method of "digiting without sorting," see Lorant $[4,5]$. The idea underlying this procedure can be explained by using the following example. Let us assume that we have to add the following column of products:

$$
\begin{aligned}
& 234 \times 24 \\
& 344 \times 32 \\
& 342 \times 33 \\
& 232 \times 22
\end{aligned}
$$

In the first column of the multiplicand the number 2 appears in line 1 and line 4 . Instead of multiplying each number separately, we add the multipliers $(24+22=46)$ and add 46 to itself (i.e., $2 \times 46=46+46$ ). This result is accumulated. Likewise, 3 appears in the first column of the second and third multiplicands. We therefore add the corresponding multipliers $(32+33=65)$ and then multiply 65 by 3 $(3 \times 65=65+65+65)$. This quantity is likewise accumulated. The first column is thus completely accounted for; the same procedure is employed for the other columns. Thus, the process of multiplication, more time-consuming than addition, has been completely replaced by summations, which can be performed on an electric accounting machine. 
TABLE 2: The values of $F_{m n}$.

\begin{tabular}{c|c||c}
\hline \hline$m$ & $n$ & $F_{m n}$ \\
\hline 0 & 0 & 27.507 \\
1 & 1 & 122.38 \\
2 & 2 & 737.97 \\
3 & 3 & $5,087.7$ \\
4 & 4 & $38,003$. \\
5 & 5 & $300,110$. \\
6 & 6 & $2,471,500$. \\
7 & 7 & $21,041,000$. \\
8 & 8 & $184,060,000$. \\
4 & 0 & -169.13 \\
5 & 1 & $-1,523.2$ \\
6 & 2 & $-13,843$. \\
7 & 3 & $-126,920$. \\
8 & 4 & $-1,174,000$. \\
8 & 0 & -760.2 \\
\hline
\end{tabular}

In Table 2 the values of $F_{m n}$ obtained in our case are given ${ }^{3}$ for $m \leqq 8, n \leqq 8$. It must be remembered that $F_{m n}=F_{n m}$ in this case (but not in general). All $F_{n m}$ with $m \leqq 8$ and $n \leqq 8$ which are not listed are equal to zero.

4. Computation of the determinants. In order to determine $\phi^{(n)}(z)$, defined by Eq. (2.1), we must compute a series of determinants whose elements are complex numbers. In this section we indicate a method for evaluating determinants with complex elements by the use of punch-card machines.

We shall again explain the procedure by means of an illustrative example, referring for detailed information to Lorant [6]. Let the determinant be:

$$
\left|\begin{array}{ccc}
a_{11}+i A_{11} & a_{12}+i A_{12} & a_{13}+i A_{13} \\
a_{21}+i A_{21} & a_{22}+i A_{22} & a_{23}+i A_{23} \\
a_{31}+i A_{31} & a_{32}+i A_{32} & a_{33}+i A_{33}
\end{array}\right|
$$

where the quantities $a$ and $A$ are real numbers.

We shall assume that none of the three complex numbers in the first row is equal to zero. We then take the reciprocal of each of the three numbers in the first row. The reciprocal of any complex number not equal to zero is given by the formula

$$
\frac{1}{a+i A}=\frac{a}{a^{2}+A^{2}}-\frac{i A}{a^{2}+A^{2}} \text {. }
$$

In this way we obtain the three reciprocals

$$
r_{k}+i R_{k}=1 /\left(a_{1 k}+i A_{1 k}\right) \quad(k=1,2,3)
$$

Now a card is punched for each of the nine elements of the determinant, the entries being given in Fig. 2, where $n$ is the number of the row and $m$ the number of the column in which the element is located. If a number is negative, we punch a hole in the corner above it. See the columns for $a_{n m}$ and $r_{n}$, where the hole is marked by an $X$. In this case the machines automatically replace addition by subtraction and, in multiplying, punch a hole if one of the factors has a hole.

Note that the entries in the last two columns will have indices running from 0 to 2 instead of from 1 to 3 , as formerly. Now disregard those cards on which either or

${ }^{3}$ The author wishes to thank Dr. Edwin L. Crow for valuable advice and assistance in the performance of numerical computations. 


\begin{tabular}{|c|c|c|c|c|c|c|c|c|c|c|c|c|}
\hline 1 & 2 & 3 & $\times 4$ & 5 & $\times 6$. & 7 & 8 & 9 & 10 & 11 & 12 & 13 \\
\hline 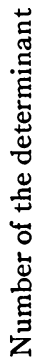 & $\approx$ & \& & 5 & $\underset{7}{\stackrel{E}{L}}$ & 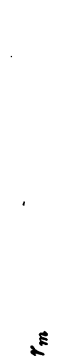 & تج & 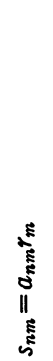 & 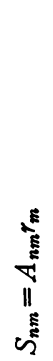 & | & 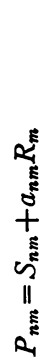 & 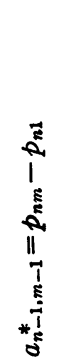 & 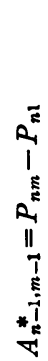 \\
\hline
\end{tabular}

FIG. 2. The entries on punch cards for the computation of a determinant. Every column represents a field on a punch card.

both of the two subscripts of $a^{*}$ and $A^{*}$ are zero; thus five of the nine cards are disregarded. From the remaining four cards we set up a new determinant

$$
\left|\begin{array}{cc}
a_{11}^{*}+i A_{11}^{*} & a_{12}^{*}+i A_{12}^{*} \\
a_{21}^{*}+i A_{21}^{*} & a_{22}^{*}+i A_{22}^{*}
\end{array}\right|
$$

Repetition of the above procedure reduces this determinant to a single element $a_{1}^{* *}+i A_{1}^{* *}$.

The value of the original determinant is then equal to

$$
\left(a_{11}+i A_{11}\right)\left(a_{12}+i A_{12}\right)\left(a_{13}+i A_{13}\right)\left(a_{11}^{*}+i A_{11}^{*}\right)\left(a_{12}^{*}+i A_{12}^{*}\right)\left(a_{11}^{* *}+i A_{11}^{* *}\right) .
$$

If we had started with a fourth-order determinant instead of a third-order one, the first stage would have reduced it to one of the third order, the second stage to one

TABLE 3: The values of $a_{n}, b_{n}$, etc.

\begin{tabular}{c||c|c|c|c|c|c}
\hline \hline$n$ & $a_{n}$ & $b_{n}$ & $c_{n}$ & $a_{n} / n$ & $b_{n} /(n-4)$ & $c_{n} /(n-8)$ \\
\hline 1 & $1.9069 \times 10^{-1}$ & - & - & $1.9069 \times 10^{-1}$ & - & - \\
2 & $9.0407 \times 10^{-2}$ & - & - & $4.5203 \times 10^{-2}$ & - & - \\
3 & $3.6806 \times 10^{-2}$ & - & - & $1.2269 \times 10^{-2}$ & - & - \\
4 & $1.4020 \times 10^{-2}$ & - & - & $3.5050 \times 10^{-3}$ & - & - \\
5 & $5.2013 \times 10^{-3}$ & $3.1980 \times 10^{-2}$ & - & $1.0403 \times 10^{-3}$ & $3.1980 \times 10^{-2}$ & - \\
6 & $1.8859 \times 10^{-3}$ & $2.3472 \times 10^{-2}$ & - & $3.1432 \times 10^{-4}$ & $1.1736 \times 10^{-2}$ & - \\
7 & $6.7240 \times 10^{-4}$ & $1.2613 \times 10^{-2}$ & - & $9.6057 \times 10^{-5}$ & $4.2043 \times 10^{-3}$ & - \\
8 & $2.3652 \times 10^{-4}$ & $5.9007 \times 10^{-8}$ & - & $2.9566 \times 10^{-5}$ & $1.4752 \times 10^{-3}$ & - \\
9 & $8.2631 \times 10^{-8}$ & $2.6348 \times 10^{-8}$ & $1.8437 \times 10^{-2}$ & $8.1812 \times 10^{-6}$ & $5.2697 \times 10^{-4}$ & $1.8437 \times 10^{-2}$ \\
\hline
\end{tabular}

of the second order, and a third stage would have been necessary to obtain a reduction to a single element. From this example it should be quite clear how to proceed in the general case.

Now

$$
\phi^{(n)}(z)=a_{n} z^{n-1}+b_{n} z^{n-5}+c_{n} z^{n-9}+\cdots
$$


and for $\psi_{n}$ and $\theta_{n}$ we have the equations

$$
\psi_{n}=\left(a_{n} / n\right) r^{n} \cos n \vartheta+\left(b_{n} / n-4\right) r^{n-4} \cos (n-4) \vartheta+\cdots
$$

and

$$
\theta_{n}=\left(a_{n} / n\right) r^{n} \sin n \vartheta+\left(b_{n} / n-4\right) r^{n-4} \sin (n-4) \vartheta+\cdots .
$$

The values of $a_{n}, b_{n}, c_{n}, a_{n} / n, b_{n} /(n-4), c_{n} /(n-8)$ are given in Table 3.

5. Determination of the function $H(r, \vartheta)$. In order to determine the harmonic

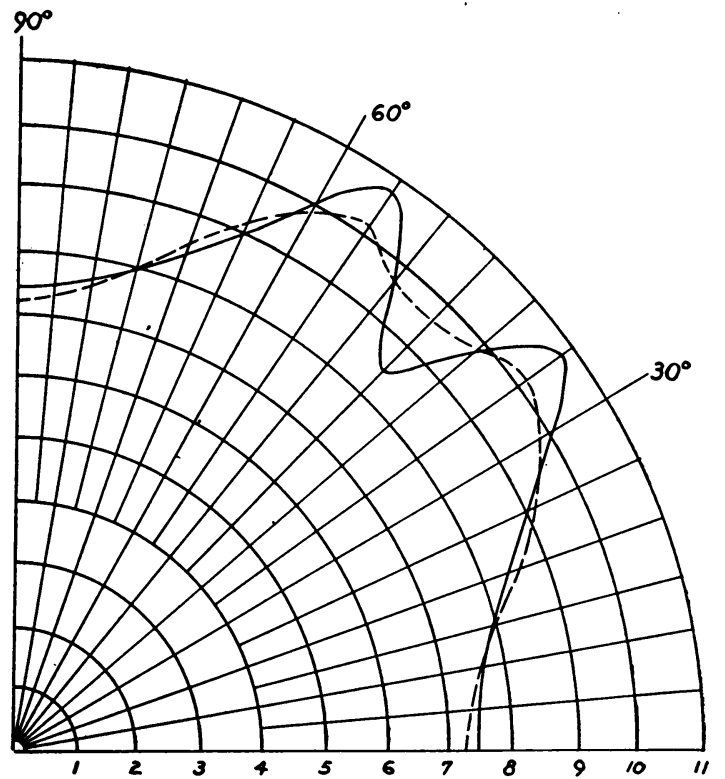

Fig. 3. The exact values of $r^{2}(\vartheta)$ along the boundary $C$ of $B$ (first quadrant) and the corresponding approximate values $H_{8}[r(\vartheta), \vartheta]$ indicated by dashed line.

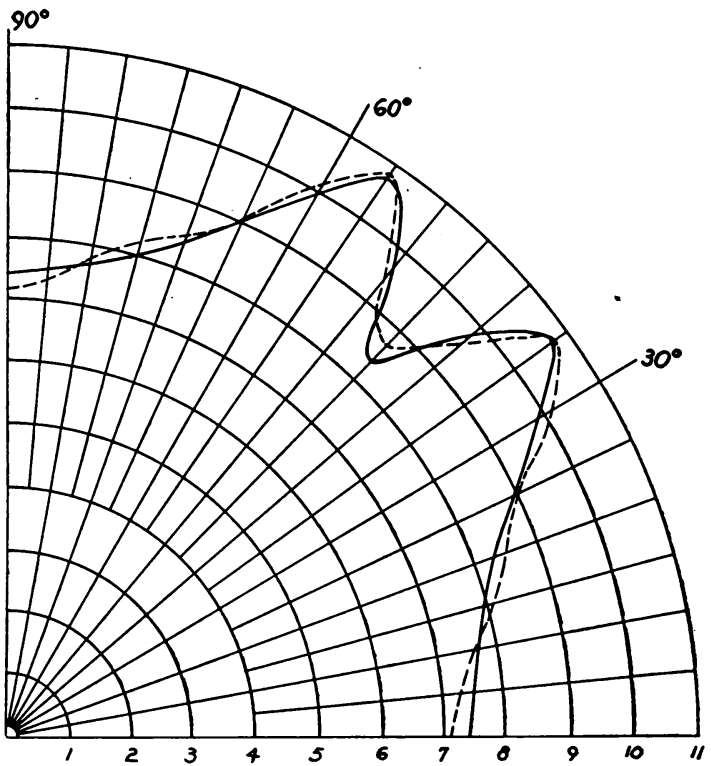

Fig. 4. The exact values of $r^{2}(\vartheta)$ along the boundary $C$ of $B$ (first quadrant) and the corresponding approximate values of $S[r(\vartheta), \vartheta]$ indicated by a dotted line.

function $H(r, \vartheta)$ which assumes the prescribed values, it remains only to determine the coefficients.

$$
A_{\nu}=\int_{C} f(s) d \theta_{\nu}(s) \quad \text { and } \quad B_{\nu}=\int_{C} f(s) d \psi_{\nu}(s) .
$$

In our case $f(s)=x_{s}^{2}+y_{s}^{2}=[r(\vartheta)]^{2}$, where $[r(\vartheta)]^{2}$ is given in column 3 of Table 1 .

The above integral representation for $A_{\nu}$ can be approximated by the summation

$$
A_{\nu}=\frac{8 \pi}{180}\left\{\frac{1}{2}\left[r\left(0^{\circ}\right)\right]^{2}+\frac{1}{2}\left[r\left(45^{\circ}\right)\right]^{2}+\sum_{\mu=1}^{44}\left[r\left(\mu^{\circ}\right)\right]^{2}\left[\theta_{\nu}\left(s_{\mu}\right)-\theta_{\nu}\left(s_{\mu-1}\right)\right]\right\}
$$

where $s_{\mu}$ is the point on $C$ having the polar angle of $\mu$ degrees.

The expression for $B_{\nu}$ is exactly the same as (5.2) except that $\theta_{\nu}$ is replaced by $\psi_{\nu}$. (It will be noted that instead of summing from $0^{\circ}$ to $360^{\circ}$ it suffices to sum from $0^{\circ}$ to $45^{\circ}$ and multiply by 8 ; this is not justified in general, but is in this particular case, 
because of the symmetry of the functions $\theta_{\nu}$ and $\psi_{\nu}$ and of the values of $r^{2}\left(=x_{s}^{2}+y_{s}^{2}\right)$ on $C$.)

The summations can be easily performed by using the "method of digiting." See $[4,5]$. Because of the symmetry of the specific problem under consideration, most of the coefficients vanish. In fact, it can easily be proven, and confirmed by computation, that all the $A_{v}$ will equal zero, while only $B_{4}, B_{8}$, etc., will differ from zero. $B_{4}$ and $B_{8}$ have been calculated and the values obtained are: $B_{4}=-4.734, B_{8}=-2.343$.

The function $H$, or more precisely the approximation $H_{8}$ obtained by taking only the first eight $\theta_{\nu}$ and $\psi_{\nu}$, is now completely determined except for the additive constant which we have designated $c_{8}$. Since the infinite sum has been replaced by a finite approximation which we may designate by $H_{8}$. it cannot be expected that $r^{2}-H_{8}$ will be absolutely constant. Therefore, the following process is employed in order to obtain an "average" value of $c_{8}$.

We evaluate $H_{8}$ on the boundary; i.e., we find $\sum_{\nu=1}^{8}\left[A_{\nu} \psi_{\nu}(s)-B_{\nu} \theta_{\nu}(s)\right]$ at a number of points on the boundary $C$. We then subtract these values from the corresponding values of $r^{2}$, and average the differences. Thus an approximate value of $c_{8}$ is ob-

TABLE 4: The values of $H_{8}(\rho, \vartheta)$ in $B$.

\begin{tabular}{r||c|c|c|c|c|c}
\hline \hline$\vartheta$ & & & & & \\
\hline $0^{\circ}$ & .5 & 1.0 & 1.5 & 2.0 & 2.5 & 3.0 \\
\hline $5^{\circ}$ & 8.491 & 8.472 & 8.389 & 8.154 & 7.603 & \\
$10^{\circ}$ & 8.491 & 8.473 & 8.395 & 8.177 & 7.675 & \\
$15^{\circ}$ & 8.491 & 8.477 & 8.414 & 8.243 & 7.874 & \\
$20^{\circ}$ & 8.491 & 8.482 & 8.442 & 8.340 & 8.153 & \\
$25^{\circ}$ & 8.492 & 8.489 & 8.476 & 8.453 & 8.455 & \\
$30^{\circ}$ & 8.492 & 8.496 & 8.511 & 8.564 & 8.727 & 9.201 \\
$35^{\circ}$ & 8.493 & 8.502 & 8.544 & 8.661 & 9.936 & 9.531 \\
$40^{\circ}$ & 8.493 & 8.507 & 8.569 & 8.735 & 9.074 & 9.657 \\
$45^{\circ}$ & 8.493 & 8.511 & 8.586 & 8.780 & 9.147 & 8.670 \\
\hline
\end{tabular}

tained, and we obtain as the best possible approximation to $H_{8}(r, \vartheta)$ the quantity

$$
c_{8}+\sum_{\nu=1}^{8}\left[A_{\nu} \psi_{\nu}(r, \vartheta)-B_{\nu} \theta_{\nu}(r, \vartheta)\right]
$$

In the example under consideration $c_{8}=8.492$.

In the column 4 of Table 1 the values of $H_{8}[r(\vartheta), \vartheta]-c_{8}$ and in column 5 those of $H_{8}[r(\vartheta), \vartheta]$ are given. The values of $H_{8}(\rho, \vartheta)$ in $B$ are given in Table 4.

6. Methods for improving the approximate solution. The solution obtained represents a rather rough approximation. ${ }^{4}$ It could naturally be improved by computing more orthogonal functions and determining more terms of the development.

${ }^{4}$ Since the aim of the present paper is to explain the procedures used rather than to obtain the best possible numerical results, the author attempted to avoid long computations and computed only very few orthogonal functions.

In actual applications of the method one will naturally determine more of these functions, in order to obtain a much better approximation. 
In many instances, however, one can save a considerable amount of labor by the use of the following simplified procedure: Suppose that there exists a domain, say $M$, which differs only slightly from $B$, see $\S 1$ and Fig. 1., and which includes $B+C$ in its interior. Let us assume further that either the function mapping $M$ into the unit circle or a complete set of orthogonal functions $\left\{f_{\mu}(z)\right\}, \mu=1,2,3, \cdots$, of $M$ are known. ${ }^{5}$ Here we choose for $M$ a circle of radius $R=3.295$.

Let $\left(r_{\nu}, \vartheta_{v}\right), \nu=1,2, \cdots, m$ denote the polar coordinates of $m$ points of the boundary $C$ of $B$, and $q$, the differences between the desired boundary values and those obtained by the use of orthogonal functions, i.e. let

$$
q_{\nu}=f\left(r_{\nu}, \vartheta_{\nu}\right)-H_{n}\left(r_{\nu}, \vartheta_{\nu}\right), \quad \nu=1,2, \cdots, m .
$$

In Table 5, Columns 2, 3 and 6 the values of $\vartheta_{v}, r_{\nu}$ and $q_{v}$, respectively are given.

TABLE 5: The values of $r(\vartheta), r^{2}(\vartheta), H_{8}\left(r_{\nu}, \vartheta_{v}\right), c\left(r_{\nu}, \vartheta_{v}\right), A\left(r_{\nu}, \vartheta_{\nu}\right)$ and $S\left(r_{\nu}, \vartheta_{\nu}\right)$ on the boundary $C$ of $B$.

\begin{tabular}{c||c|c|r|r|r|r|r|r}
\hline \hline$\nu$ & $\vartheta_{\nu}$ & $r_{\nu}$ & \multicolumn{1}{c|}{$r_{\nu}^{2}$} & $H_{8}\left(r_{\nu}, \vartheta_{\nu}\right)$ & \multicolumn{1}{c|}{$q_{\nu}$} & $c\left(r_{\nu}, \vartheta_{\nu}\right)$ & $A\left(r_{\nu}, \vartheta_{\nu}\right)$ & $S\left(r_{\nu}, \vartheta_{\nu}\right)$ \\
\hline \multirow{2}{*}{1} & 0 & 2.72 & 7.41 & 7.18 & 0.23 & 0.4 & -0.02 & 7.16 \\
2 & $10^{\circ}$ & 2.76 & 7.64 & 7.56 & 0.10 & 0.5 & 0.07 & 7.63 \\
3 & $20^{\circ}$ & 2.90 & 8.39 & 8.57 & -0.18 & 1.0 & -0.09 & 8.48 \\
4 & $25^{\circ}$ & 3.00 & 9.00 & 9.20 & -0.20 & 2.0 & -0.19 & 9.01 \\
5 & $30^{\circ}$ & 3.14 & 9.88 & 9.80 & 0.08 & 0.9 & 0.32 & 10.12 \\
6 & $35^{\circ}$ & 3.29 & 10.84 & 10.13 & -0.59 & & 0.69 & 10.82 \\
7 & $40^{\circ}$ & 3.11 & 9.69 & 9.79 & -0.10 & 0.2 & -0.33 & 9.46 \\
8 & $45^{\circ}$ & 2.90 & 8.49 & 9.57 & -1.14 & 0.5 & -0.98 & 8.59 \\
\hline
\end{tabular}

If $M$ is a circle of radius $R$ we introduce the functions

$$
p(R, \tau ; r, \vartheta)=\frac{1}{2 \pi} \cdot \frac{R^{2}-r^{2}}{R^{2}+r^{2}-2 r R \cos (\vartheta-\tau)}
$$

where are harmonic for every value of $\tau$, and we determine $l$ real constants $T_{\mu}$, $\mu=1,2, \cdots, l, l \geqq m$, so that, at the points $\left(r_{v}, \vartheta_{v}\right)$, the expression

$$
A(r, \vartheta)=\sum_{\mu=1}^{l} T_{\mu} p\left(R, \tau_{\mu} ; r, \vartheta\right)
$$

equals $q_{p}$; i.e. so that

$$
\sum_{\mu=1}^{l} T_{\mu} p\left(R, \tau_{\mu} ; r_{\nu}, \vartheta_{\nu}\right)=q_{\nu}, \quad \nu=1,2, \cdots, m .
$$

Remark. The conjugate $g(R, \tau ; r, \vartheta)$ of $p(R, \tau ; r, \vartheta)$ is given by

$$
g(R, \tau ; r, \vartheta)=\frac{1}{\pi} \frac{r R \sin (\vartheta-\tau)}{R^{2}-2 r R \cos (\vartheta-\tau)+r^{2}}
$$

5 The function $w(z, \bar{t})$ which maps $M$ into the unit circle (taking a point $t$ into the origin) is determined, if the set $\left\{f_{\mu}(z)\right\}$ is known, since according to $[1$, p. 53]

$$
w(z, t)=\left\{\int_{0}^{s}\left[\sum_{\mu=1}^{\infty} f_{\mu}(s) \overline{f_{\mu}(t)}\right] d z\right\}\left[\sum_{\mu=1}^{\infty}\left|f_{\mu}(t)\right|^{2}\right]^{-1 / 2}
$$


The determination of the quantities $T_{\mu}$ involves solving a system of $m$ linear equations with $n$ variables. This can be performed using punch card machines. The expression

$$
S(r, \vartheta)=H_{n}(r, \vartheta)+A(r, \vartheta)
$$

can be considered as a second approximation to the desired solution.

We wish to add that at the points $\left(R, \tau_{\mu}\right)$, where the difference $R-r_{\nu}$ becomes very small (e.g. for $\tau_{\mu}=35^{\circ}$ in our example), one has to replace $p\left(R, \tau_{\mu} ; r, \vartheta\right)$ by

$$
\omega\left(R, \tau_{\mu}^{(0)}, \tau_{\mu}^{(1)} ; r, \vartheta\right)=\int_{\tau_{\mu}(0)}^{\tau_{\mu}(1)} p(R, \tau ; r, \vartheta) d \tau,
$$

where $\tau_{\mu}^{(0)} \tau_{\mu}^{(1)}\left(\tau_{\mu}^{(0)}<\tau_{\mu}<\tau_{\mu}^{(1)}\right)$ are suitably chosen quantities.

We note that the expression (6.3) can be considered as an approximation of the integral

$$
\int_{0}^{2 \pi} p(R, \tau ; r, \vartheta) h(\tau) d \tau=\frac{1}{2 \pi} \int_{0}^{2 \pi} \frac{\left(R^{2}-r^{2}\right) h(\tau) d \tau}{R^{2}+r^{2}-2 r R \cos (\vartheta-\tau)}
$$

where $h(\tau)$ is some continuous function which at the points $\tau_{\mu}$ assumes the values $\left[T_{\mu} /\left(\tau_{\mu}^{(0)}-\tau_{\mu}^{(1)}\right)\right]$. In many instances instead of solving Eq. (6.4), we can estimate the values of $T_{\mu}$, taking $T_{\mu}$ approximately equal to $q_{v}$.

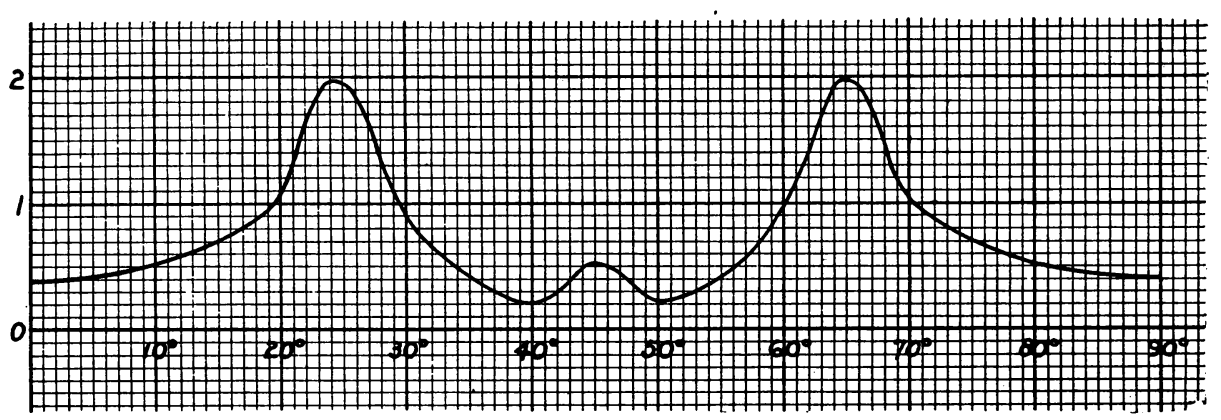

FIG. 5. The values of $\sum_{k=0}^{3}\left[p\left(R, 20^{\circ}+k \cdot 90^{\circ} ; r(\vartheta), \vartheta\right)+p\left(R, 70^{\circ}+k \cdot 90^{\circ} ; r(\vartheta), \vartheta\right)\right]$, considered as function of $\vartheta$, along the boundary $C$ of $B$.

In the example under consideration we took for ${ }^{7} A(r, \vartheta)$,

$$
\begin{aligned}
A(r, \vartheta) & -0.2 \sum_{k=0}^{3}\left[p\left(R, 25^{\circ}+k \cdot 90^{\circ} ; r, \vartheta\right)+p\left(R, 65^{\circ}+k \cdot 90^{\circ} ; r, \vartheta\right)\right] \\
& +0.706 \sum_{k=0}^{3}\left[p\left(R, 35^{\circ}+k \cdot 90^{\circ} ; r, \vartheta\right)+p\left(R, 55^{\circ}+k \cdot 90^{\circ} ; r, \vartheta\right)\right] \\
& -1.139 \sum_{k=0}^{3} p\left(R, 45^{\circ}+k \cdot 90^{\circ} ; r, \vartheta\right), \\
R= & 3.295 .
\end{aligned}
$$

${ }^{7}$ We note that since $r\left(35^{\circ}\right)$ is almost equal to 3.295, we make an over-simplification by replacing $0.706\left[\left(\tau_{6}^{(1)}-\tau_{6}^{(0)}\right) \omega\left(R, \tau_{6}^{(1)}, \tau_{6}^{(0)} ; r, \vartheta\right)+\cdots\right]$ by $0.706\left[p\left(R, 35^{\circ}, r, \vartheta\right)+\cdots\right]$;

this approximation, although valid approximately for points which are far enough away from $\left(R, 35^{\circ}+k \cdot 90^{\circ}\right),\left(R, 55^{\circ}+k \cdot 90^{\circ}\right), k=0,1,2,3$, would have to be replaced by more exact expressions in the neighborhood of these points. 
The values of

$$
c(r, \vartheta)=\sum_{k=0}^{3}\left[p\left(R, 25^{\circ}+k \cdot 90^{\circ} ; r, \vartheta\right)+p\left(R, 65^{\circ}+k \cdot 90^{\circ} ; r, \vartheta\right)\right]
$$

at points $\left(r_{\nu}, \vartheta_{\nu}\right)$ are given in column 7 of Table 5 and drawn in Fig. 5.

The values of $A(r, \vartheta)$ and $S(r, \vartheta)$ are indicated in columns 8 and 9 of Table 5. In the case where $M$ is not a circle, but the mapping function of $M$ into the unit circle is known, we can proceed similarly. A very convenient procedure which can be applied in this case consists in the use of curvilinear coordinates (in $G$ ) which correspond to polar coordinates in the circle.

On the other hand, if a complete system of orthogonal functions $\left\{f_{\mu}\right\}, \mu=1,2, \cdots$ of the domain $M$ is known, we can construct in $M$ a set of functions $P_{\mu}$ which possess properties similar to the functions $p\left(R, \tau_{\mu} ; r, \vartheta\right)$.

The characteristic property of the function $p(R, \tau ; r, \vartheta)$ is that it is harmonic in $M$, it becomes infinite at the point $(r, \vartheta)=(R, \tau)$ and vanishes on the remaining part of the boundary of $M$.

We denote by $P_{\mu}$ a system of functions which approximately equal unity on a small interval of the boundary and zero on the rem aining part. Using the system $\left\{f_{v}\right\}$ of orthogonal functions, we can determine a set of functions $P_{\mu}$, and then proceed as before, replacing the $p\left(R, \tau_{\mu} ; r, \vartheta\right)$ by the $P_{\mu}$ 's.

7. Determination of the stresses. The determination of the stresses requires the evaluation of the derivative of $H$. Our methods yield only functions $H_{N}$ and $S$, approximating the required function. Inside the domain $B$ the derivatives $\left(\partial H_{N} / \partial x\right.$, $\left.\partial H_{N} / \partial y\right)$ and $(\partial S / \partial x, \partial S / \partial y)$ will in general approximate the corresponding exact solution quite satisfactorily. The evaluation of these derivatives may proceed by use of punch-card machines, in the manner described previously. Near the boundary or on the boundary itself the approximations obtained for the derivatives will, in many instances, not be satisfactory, and it is advisable to use some summation method in order to obtain more exact values.

In particular at sharp edges, or points where the radius of curvature of the boundary is no longer continuous, it is necessary to apply special methods for the evaluation of the derivatives. These questions require special treatment, and will be discussed in another paper.

8. Additional remarks. It should be noted that the method described in the present paper can be extended without difficulty to the study of thin plates as well as to the general problem of elasticity in three dimensions (see [8]).

Up to the present time, it has been practically impossible to determine Green's function for the equation $\Delta u=0$ in the case of multiply-connected domains. In a recent paper [9] Schiffer has indicated a simple relation between Green's function of Laplace's equations and systems of orthonormal functions which permits a comparatively simple determination of Green's function of such domains.

Finally by introducing some additional considerations the method of orthogonal functions can be extended to a large class of linear partial differential equations in two and three variables (see [10], [11]). 


\section{REFERENCES}

1. S. Bergman, Partial differential equations (mimeographed lecture notes), Brown University, Providence 1941.

2. S. Bergman, Sur les fonctions orthogonales, Interscience Publishers, New York, 1941.

3. W. J. Eckert, Punched card methods in scientific computation, Watson Computing Bureau, Columbia University, 1940.

4. R. Lorawt, Digiting without sorting, Pointers, International Business Machines Corporation, Group 9, No. 461.

5. R. Lorant, Sum of products and squares by card cycle total transfer, Pointers, International Business Machines Corporation, Group 9, No. 478.

6. R. Lorant, Evaluation of the determinants, Pointers, International Business Machines Corporation, Group 9, (to be published soon).

7. A. E. H. Love, A treatise on the mathematical theory of elasticity, Dover Publications, New York, 1944.

8. S. Bergman, Über die Bestimmung der elastischen Spannungen und Verschiebungen in einem konvexen Körper, Math. Annalen, 98, 248-263 (1927).

9. M. Schiffer, On the kernel of orthonormal systems, Duke Math. J. 13, 529-540 (1946).

10. S. Bergman, Zur Theorie der Funktionen die eine lineare partielle Differentialgleichung befriedigen, Reccueil Math. 2, 1169-1198 (1937).

11. S. Bergman, On functions satisfying certain classes of partial differential equations'of elliptic type and their representation, (to.be published soon). 